\title{
Editorial: The Emerging Role of Artificial Intelligence in Dermatology
}

\author{
Farhan Mahmood ${ }^{1}$, Solomon Bendayan ${ }^{2}$, Feras M. Ghazawi ${ }^{3}$ and Ivan V. Litvinov ${ }^{4 *}$ \\ ${ }^{1}$ Faculty of Medicine, University of Ottawa, Ottawa, ON, Canada, ${ }^{2}$ Faculty of Medicine, McGill University, Montréal, QC, \\ Canada, ${ }^{3}$ Division of Dermatology, University of Ottawa, Ottawa, ON, Canada, ${ }^{4}$ Division of Dermatology, McGill University, \\ Montréal, QC, Canada
}

Keywords: artificial intelligence, machine learning, deep learning, dermatology, teledermatology, COVID-19

\section{Editorial on the Research Topic}

\section{The Emerging Role of Artificial Intelligence in Dermatology}

The use of artificial intelligence (AI) in dermatology is an emerging area of interest with several applications highlighted in the special topic, including the differentiation of benign and malignant pigmented lesions, improvement of diagnosis and management of psoriasis and other inflammatory diseases, assessing ulcer specifications, and gene expression profiling Gomolin et al.. Over the past decades, there has been a significant focus in analyzing and classifying data from skin lesions using machine learning (ML) models $(1,2)$. In this editorial, we highlight previous and recent applications of AI and its use during the coronavirus disease 2019 (COVID-19) pandemic.

It was previously illustrated that $\mathrm{AI}$ is able to distinguish between benign nevi vs. melanoma using individual pixels from dermatoscopic and non-dermatoscopic images (3-19). Jutzi et al. further assessed the attitudes of patients toward AI and demonstrated that most respondents supported the use of AI, particularly to help detect melanoma early at home. However, potential errors, poor/inconsistent image quality and insufficient data protection of AI still pose important barriers. Recently, ML and convolutional neural network (CNN) models that classify melanoma on histopathological or clinical images demonstrated ability to achieve exceedingly high sensitivities and diagnostic accuracies (20-24). ML models are also being trained using substantial data sets including more racially diverse data, making AI more accessible for use in remote and resourcelimited healthcare settings $(20,25)$. There is also a rise in smartphone applications with classifying the risk of photographed lesions or detecting malignant/premalignant lesions on histopathological images $(26,27)$. One study assessed whether dermatoscopic or reflectance confocal microscopy (RCM) findings correlated with histologic diagnoses of melanocytic lesions with peripheral globules (28). They found that dermoscopy and RCM diagnosed $100 \%$ of melanomas and $84.21 \%$ of dysplastic nevi accurately. Dysplastic nevi and melanocytic lesions differed significantly based on the sizes and shapes of peripheral globules, and the signs of malignancy on RCM including pagetoid cells, non-edged papillae, atypical junctional thickenings, and atypical cells at the dermal-epidermal junction. A top-ranked computer algorithm has also been shown to classify images of melanomas, nevi, and seborrheic keratoses with a higher specificity than dermatologists (85.0 vs. $72.6 \%$ ) (29). Thus, AI has the potential to classify skin images of melanoma and its benign mimickers with high accuracy.

However, despite the increased accuracy of diagnosing melanomas, clinicians or AI cannot reliably predict the oncologic transformation of nevi (30). This is due to the static nature of the nevi on clinical presentation, and the lack of data to train AI on the evolution of certain melanocytic nevi including dysplastic or spitzoid nevi. Further research is required to understand how AI can identify the progression of dysplastic nevi. 
Gomolin et al. and Schäfer et al. both highlighted the use of AI in ulcer assessment. Schäfer et al. demonstrated that predictive values including low household income, older age, and comorbidities were associated with higher risks for diabetic foot ulcer (DFU) and amputation. However, ML models did not achieve reliable results when predicting the prognosis of DFUs or amputations using the predictive values. The authors highlighted the development of models that can detect wound progression of DFUs using predictive values as next steps Schäfer et al.. Recently, $\mathrm{CNN}$ models have emerged categorizing wound images based on their etiology (31) and are being implemented in smartphone application to detect DFU wounds with high inter- and intraobserver reliabilities compared to traditional measurements (32). Notably, the combination of clinical laboratory (e.g., glomerular filtration rate) data from health records and image features of patients with DFUs by a ML model predicted the healing of DFUs, with reliable accuracies (33). Polarized hyperspectral imaging and ML technology has also recently shown to be a potential avenue to characterize detailed pathological complications of ulcers (34).

Similarly, a review by $\mathrm{Du}$ et al. illustrated that ML has the potential to predict the clinical outcomes and prognosis of several dermatoses. Larger sample sizes of data enable ML algorithms to produce accurate outputs; however, this may be a limitation since national and international collaborations between registries are required to acquire large dermatologic data sets. The authors underscore the need for prospective clinical trials to validate the use of ML models to predict outcomes. Recently, a ML model accurately predicted the Dermatology Quality of Life Index for patients with psoriasis withdrawing from risankizumab (35), while similar ML technology was used to build a highly accurate biomarker predicting the progression of alopecia areata to alopecia totalis or alopecia universalis (36). A multicenter prospective open label pilot study was recently conducted treating psoriasis patients with secukinumab. A predictive model was developed using clinical attributes of patients, achieving an accuracy of $91.88 \%$ in predicting responders and nonresponders (37).

A recent study by Showalter et al. (38) shed light onto the potential application of AI to inflammatory dermatology conditions. Showalter et al. aimed to determine the histologic and gene expression features of clinical improvement in early diffuse cutaneous systemic sclerosis (dcSSc) by evaluating skin biopsies from patients with dcSSc. They used support vector machine learning using scleroderma gene expression subset as classifiers and histology scores as inputs. In the samples with the highest Modified Rodnan Skin Score, alpha-smooth muscle actin (ASMA) was the highest and CD34 was the lowest, and these markers were the strongest predictors of gene expression subset. CD34 staining was the highest in the normal subset and ASMA was highest in the inflammatory subset. The CD34 and ASMA binarized scores also identified a 47-gene fibroblast polarization signature which decreased in patients with clinical improvement compared to those with no improvement. Systemic sclerosis histologic features have been shown to correlate with the Modified Rodnan Skin Score; however, this recent study highlights the potential to use dermal fibroblast polarization between aSMA and CD34 to describe clinical improvement for dcSSc.

Heckler et al. previously examined the effects of label noise on the performance of CNN models when classifying skin cancers. They found that ML models are highly sensitive to label noise, highlighting the need for biopsy-verified images to train models. Recently, a hybrid evolutionary optimization technique based on two swarm intelligence algorithms was alternatively proposed to overcome the premature local convergence of single conventional clustering algorithms when detecting and segmenting psoriasis lesions $(39,40)$. Global convergence of algorithms has demonstrated superiority over conventional clustering techniques $(39,40)$, revealing the potential to combine multiple algorithms to overcome the biases and restrictions of single models.

Importantly, the social distancing restrictions imposed by the COVID-19 pandemic fostered digital transformations in dermatology and sparked COVID-19-specific applications of AI and technology. For instance, several cutaneous manifestations are associated with COVID-19 including pernio-like and vesicular lesions (41); CNN models are being developed to detect these lesions in patients with Fitzpatrick I-VI skin, further optimizing contact-free healthcare $(42,43)$.

During the pandemic, there has also been an increased uptake of teledermatology worldwide to ensure patients and healthcare providers have access to dermatologists (44-46). Teledermatology enables patients from remote/rural areas and/or underserved populations to obtain access, while reducing wait times for dermatology referrals (47-49). Recently, ML models have been developed to assess the quality of photos submitted by patients to teledermatology consults-rejecting low quality and retaining high quality photographs (50). ML models are now being used to classify dermatology clinical images in the electronic health records to optimize access to these images for research purposes (51). Furthermore, recent studies have demonstrated that dermoscopy improves the diagnostic accuracy of teledermatology (52); thus, smartphone microscope applications employing $\mathrm{CNN}$ are now increasingly supplementing teledermatology consults (53). Furthermore, because several dermatoses are usually initially seen by primary care providers (PCP), an AI-based tool was developed to interpret images and their associated medical histories, which improved the diagnoses of dermatoses by PCPs in a teledermatology setting (54).

Social media has also become an important component of technology and dermatology during the COVID-19 pandemic. The use of social media in dermatology increased over the recent years and common uses for dermatologists include information dissemination, knowledge sharing, and networking (55-57). Thus, social media enables knowledge-exchange and interactivity during the pandemic-when access to dermatologists may be restricted.

Although there are several applications of AI in dermatology, there are certain barriers preventing its uptake. With respect to teledermatology, full-body skin examinations are difficult to perform virtually, thus, clinically significant lesions may be missed $(58,59)$. Several costs are also associated with 
the implementation of teledermatology (i.e., equipment costs, technological competencies, and staff training), while ensuring encryption to protect confidential medical data (60-62). Teledermatology may also be unavailable for individuals who do not have access to high quality internet or telecommunication devices with high quality cameras (63).

Several other barriers exist with regards to the implementation of AI in clinical dermatology, which have been extensively discussed by Gomolin et al. as well, including generalizability, standardization, and interpretability (3, 64-67). To summarize, several AI algorithms are trained using input data from limited populations, thus they may not be effective in patients from different settings or with unique phototypes. Universal quality standards for images used to train AI or used for dermoscopy are also lacking. Further, patients appreciate the traditional encounter with the physician and the accountability that it entails. Physicians alike, are resistant to accept that AI can

\section{REFERENCES}

1. Hogarty DT, Mackey DA, Hewitt AW. Current state and future prospects of artificial intelligence in ophthalmology: a review. Clin Exp Ophthalmol. (2019) 47:128-39. doi: 10.1111/ceo.13381

2. Vestergaard ME, Menzies SW. Automated diagnostic instruments for cutaneous melanoma. Semin Cutan Med Surg. (2008) 27:32-6. doi: 10.1016/j.sder.2008.01.001

3. Gomolin A, Netchiporouk E, Gniadecki R, Litvinov IV. Artificial intelligence applications in dermatology: where do we stand? Front Med. (2020) 7:100. doi: 10.3389/fmed.2020.00100

4. Xie F, Fan H, Li Y, Jiang Z, Meng R, Bovik A. Melanoma classification on dermoscopy images using a neural network ensemble model. IEEE Trans Med Imaging. (2017) 36:849-58. doi: 10.1109/TMI.2016.2633551

5. Yu C, Yang S, Kim W, Jung J, Chung KY, Lee SW, Oh B. Acral melanoma detection using a convolutional neural network for dermoscopy images. PLoS ONE. (2018) 13:e0193321. Erratum in: PLoS ONE. (2018) 13:e0196621. doi: 10.1371/journal.pone.0196621

6. Pour MP, Seker H. Ling Shao. Automated lesion segmentation and dermoscopic feature segmentation for skin cancer analysis. Annu Int Conf IEEE Eng Med Biol Soc. (2017) 2017:640-3. doi: 10.1109/EMBC.2017.8036906

7. Jafari MH, Samavi S, Karimi N, Soroushmehr SM, Ward K, Najarian K. Automatic detection of melanoma using broad extraction of features from digital images. Annu Int Conf IEEE Eng Med Biol Soc. (2016) 2016:135760. doi: 10.1109/EMBC.2016.7590959

8. Bi L, Kim J, Ahn E, Kumar A, Fulham M, Feng D. Dermoscopic image segmentation via multistage fully convolutional networks. IEEE Trans Biomed Eng. (2017) 64:2065-74. doi: 10.1109/TBME.2017.2712771

9. García Arroyo JL, García Zapirain B. Detection of pigment network in dermoscopy images using supervised machine learning and structural analysis. Comput Biol Med. (2014) 44:144-57. doi: 10.1016/j.compbiomed.2013.11.002

10. Jafari MH, Nasr-Esfahani E, Karimi N, Soroushmehr SMR, Samavi S, Najarian K. Extraction of skin lesions from non-dermoscopic images for surgical excision of melanoma. Int J Comput Assist Radiol Surg. (2017) 12:102130. doi: 10.1007/s11548-017-1567-8

11. Lingala $M$, Stanley RJ, Rader RK, Hagerty J, Rabinovitz HS, Oliviero $\mathrm{M}$, et al. Fuzzy logic color detection: Blue areas in melanoma dermoscopy images. Comput Med Imaging Graph. (2014) 38:403-10. doi: 10.1016/j.compmedimag.2014.03.007

12. Souza S, Abe JM. Nevus and melanoma paraconsistent classification. Stud Health Technol Inform. (2014) 207:244-50. doi: 10.1007/978-3-319-19722-7_9

13. Tschandl P, Kittler H, Argenziano G, A. pretrained neural network shows similar diagnostic accuracy to medical students in categorizing dermatoscopic diagnose and manage dermatoses more reliably than them. Finally, most original research studies in AI have not studied the applications in large-scale clinical trials. More high-quality clinical studies are needed to substantiate the use of AI in dermatology.

In conclusion, significant advances have been made to enable AI use in dermatology. Such advancements play essential roles in detecting, diagnosing, classifying, and prognosticating dermatoses. This field will continue to evolve with the focus on improving the diagnostic accuracy of ML models, determining the use of predictive models through prospective trials, and developing smartphone applications to optimize virtual healthcare.

\section{AUTHOR CONTRIBUTIONS}

All authors listed have made a substantial, direct and intellectual contribution to the work, and approved it for publication.

images after comparable training conditions. Br J Dermatol. (2017) 177:8679. doi: 10.1111/bjd.15695

14. Afifi S, GholamHosseini H, Sinha R, SVM. classifier on chip for melanoma detection. Annu Int Conf IEEE Eng Med Biol Soc. (2017) 2017:2704. doi: 10.1109/EMBC.2017.8036814

15. Han SS, Kim MS, Lim W, Park GH, Park I, Chang SE. Classification of the clinical images for benign and malignant cutaneous tumors using a deep learning algorithm. J Invest Dermatol. (2018) 138:152938. doi: 10.1016/j.jid.2018.01.028

16. Kefel S, Pelin Kefel S, LeAnder RW, Kaur R, Kasmi R, Mishra NK, et al. Adaptable texture-based segmentation by variance and intensity for automatic detection of semitranslucent and pink blush areas in basal cell carcinoma. Skin Res Technol. (2016) 22:412-22. doi: 10.1111/srt.12281

17. Cheng B, Joe Stanley R, Stoecker WV, Osterwise CT, Stricklin SM, Hinton KA, et al. Automatic dirt trail analysis in dermoscopy images. Skin Res Technol. (2013) 19:e20-6. doi: 10.1111/j.1600-0846.2011.00602.x

18. Chang WY, Huang A, Yang CY, Lee CH, Chen YC, Wu TY, Chen GS Computer-aided diagnosis of skin lesions using conventional digital photography: a reliability and feasibility study. PLoS ONE. (2013) 8:e76212. doi: 10.1371/journal.pone.0076212

19. Guvenc P, LeAnder RW, Kefel S, Stoecker WV, Rader RK, Hinton KA, et al. Sector expansion and elliptical modeling of blue-gray ovoids for basal cell carcinoma discrimination in dermoscopy images. Skin Res Technol. (2013) 19:e532-6. doi: 10.1111/srt.12006

20. Ningrum DNA, Yuan SP, Kung WM, Wu CC, Tzeng IS, Huang CY Li JY, et al. Deep learning classifier with patient's metadata of dermoscopic images in malignant melanoma detection. J Multidiscip Healthc. (2021) 14:87785. doi: 10.2147/JMDH.S306284

21. Hekler A, Utikal JS, Enk AH, Solass W, Schmitt M, Klode J, et al. Deep learning outperformed 11 pathologists in the classification of histopathological melanoma images. Eur J Cancer. (2019) 118:916 doi: 10.1016/j.ejca.2019.06.012

22. Han SS, Moon IJ, Lim W, Suh IS, Lee SY, Na JI, et al. Keratinocytic skin cancer detection on the face using region-based convolutional neural network. JAMA Dermatol. (2020) 156:29-37. doi: 10.1001/jamadermatol.2019. 3807

23. Brinker TJ, Hekler A, Enk AH, Klode J, Hauschild A, Berking C, et al. A convolutional neural network trained with dermoscopic images performed on par with 145 dermatologists in a clinical melanoma image classification task. Eur J Cancer. (2019) 111:148-54. doi: 10.1016/j.ejca.2019.02.005

24. Brinker TJ, Hekler A, Enk AH, Klode J, Hauschild A, Berking C, et al. Deep learning outperformed 136 of 157 dermatologists in a head-to-head dermoscopic melanoma image classification task. Eur J Cancer. (2019) 113:4754. doi: 10.1016/j.ejca.2019.04.001 
25. Aggarwal SLP, Papay FA. Artificial Intelligence Image Recognition Of Melanoma And Basal Cell Carcinoma In Racially Diverse Populations. J Dermatolog Treat. (2021) 22:1-17. doi: 10.1080/09546634.2021.194 4970

26. Udrea A, Mitra GD, Costea D, Noels EC, Wakkee M, Siegel DM, et al. Accuracy of a smartphone application for triage of skin lesions based on machine learning algorithms. J Eur Acad Dermatol Venereol. (2020) 34:64855. doi: $10.1111 /$ jdv.15935

27. Sangers TE, Nijsten T, Wakkee M. Mobile health skin cancer risk assessment campaign using artificial intelligence on a population-wide scale: a retrospective cohort analysis. J Eur Acad Dermatol Venereol. (2021) 35:e7724. doi: $10.1111 /$ jdv.17442

28. Pampín-Franco A, Gamo-Villegas R, Floristán-Muruzábal U, PinedoMoraleda FJ, Pérez-Fernández E, López-Estebaranz JL. Melanocytic lesions with peripheral globules: results of an observational prospective study in 154 high-risk melanoma patients under digital dermoscopy follow-up evaluated with reflectance confocal microscopy. J Eur Acad Dermatol Venereol. (2021) 35:1133-42. doi: 10.1111/jdv.17105

29. Marchetti MA, Liopyris K, Dusza SW, Codella NCF, Gutman DA, Helba $\mathrm{B}$, et al. Computer algorithms show potential for improving dermatologists' accuracy to diagnose cutaneous melanoma: Results of the International Skin Imaging Collaboration 2017. J Am Acad Dermatol. (2020) 82:6227. doi: $10.1016 /$ j.jaad.2019.07.016

30. Sondermann W, Utikal JS, Enk AH, Schadendorf D, Klode J, Hauschild A, et al. Prediction of melanoma evolution in melanocytic nevi via artificial intelligence: a call for prospective data. Eur J Cancer. (2019) 119:304. doi: 10.1016/j.ejca.2019.07.009

31. Rostami B, Anisuzzaman DM, Wang C, Gopalakrishnan S, Niezgoda J, Yu Z. Multiclass wound image classification using an ensemble deep CNN-based classifier. Comput Biol Med. (2021) 134:104536. doi: 10.1016/j.compbiomed.2021.104536

32. Chan KS, Chan YM, Tan AHM, Liang S, Cho YT, Hong Q, et al. Clinical validation of an artificial intelligence-enabled wound imaging mobile application in diabetic foot ulcers. Int Wound J. (2021). doi: 10.1111/iwj. 13603

33. Kim RB, Gryak J, Mishra A, Cui C, Soroushmehr SMR, Najarian $\mathrm{K}$, et al. Utilization of smartphone and tablet camera photographs to predict healing of diabetes-related foot ulcers. Comput Biol Med. (2020) 126:104042. doi: 10.1016/j.compbiomed.2020.104042

34. Dremin V, Marcinkevics Z, Zherebtsov E, Popov A, Grabovskis A, Kronberga $\mathrm{H}$, et al. Skin complications of diabetes mellitus revealed by polarized hyperspectral imaging and machine learning. IEEE Trans Med Imaging. (2021) 40:1207-16. doi: 10.1109/TMI.2021.3049591

35. Papp KA, Soliman AM, Done N, Carley C, Lemus Wirtz E, Puig L. Deterioration of health-related quality of life after withdrawal of risankizumab treatment in patients with moderate-to-severe plaque psoriasis: a machine learning predictive model. Dermatol Ther. (2021) 11:1291304. doi: 10.1007/s13555-021-00550-8

36. Zhang $\mathrm{T}$, Nie Y. Prediction of the risk of alopecia areata progressing to alopecia totalis and alopecia universalis: biomarker development with bioinformatics analysis and machine learning. Dermatology. (2021) 18:111. doi: $10.1159 / 000515764$

37. Damiani G, Conic RRZ, Pigatto PDM, Carrera CG, Franchi C, Cattaneo A, et al. Predicting secukinumab fast-responder profile in psoriatic patients: advanced application of artificial-neural-networks (ANNs). J Drugs Dermatol. (2020) 19:1241-6. doi: 10.36849/JDD.2020.5006

38. Showalter K, Spiera R, Magro C, Agius P, Martyanov V, Franks $\mathrm{JM}$, et al. Machine learning integration of scleroderma histology and gene expression identifies fibroblast polarisation as a hallmark of clinical severity and improvement. Ann Rheum Dis. (2021) 80:228-37. doi: 10.1136/annrheumdis-2020-217840

39. Dash M, Londhe ND, Ghosh S, Raj R, Sonawane R. Psoriasis lesion detection using hybrid seeker optimization based image clustering. Curr Med Imaging. (2021). doi: 10.2174/1573405617666210224112123

40. Dash M, Londhe ND, Ghosh S, Shrivastava VK, Sonawane RS. Swarm intelligence based clustering technique for automated lesion detection and diagnosis of psoriasis. Comput Biol Chem. (2020) 86:107247. doi: 10.1016/j.compbiolchem.2020.107247
41. Murrell DF, Arora G, Rudnicka L, Kassir M, Lotti T, Goldust M, et al. dermatologist's perspective of the COVID-19 outbreak. Dermatol Ther. (2020) 33:e13538. doi: 10.1111/dth.13538

42. Mathur J, Chouhan V, Pangti R, Kumar S, Gupta S, A. convolutional neural network architecture for the recognition of cutaneous manifestations of COVID-19. Dermatol Ther. (2021) 34:e14902. doi: 10.1111/dth.14902

43. Sadoughifar R, Goldust $M$, Abdshahzadeh $H$, Abrishamchi $R$, Rudnicka L, Jafferany $M$, et al. Artificial intelligence in diagnosis and management of COVID-19 in dermatology. Dermatol Ther. (2020) 33:e13794. doi: 10.1111/dth.13794

44. Edwards HA, Shen X, Soyer HP. Teledermatology Adaptations in the COVID19 Era. Front Med. (2021) 8:675383. doi: 10.3389/fmed.2021.675383

45. Chow A, Teo SH, Kong JW, Lee SBM, Heng YK, van Steensel MAM, et al. Teledermatology in primary care in singapore: experiences of family doctors and specialists. Acta Derm Venereol. (2021) 101:adv00540. doi: 10.2340/00015555-3847

46. Hamad J, Fox A, Kammire MS, Hollis AN, Khairat S. Evaluating the experiences of new and existing teledermatology patients during the COVID-19 pandemic: cross-sectional survey study. JMIR Dermatol. (2021) 4:e25999. doi: 10.2196/25999

47. Woodley A. Can teledermatology meet the needs of the remote and rural population? Br J Nurs. (2021) 30:574-9. doi: 10.12968/bjon.2021.30.10.574

48. Linggonegoro D, Rrapi R, Ashrafzadeh S, McCormack L, Bartenstein D, Hazen TJ, et al. Continuing patient care to underserved communities and medical education during the COVID-19 pandemic through a teledermatology student-run clinic. Pediatr Dermatol. (2021) 38:977-9. doi: 10.1111/pde.14653

49. McAfee JL, Vij A, Warren CB. Store-and-forward teledermatology improves care and reduces dermatology referrals from walk-in clinics: a retrospective descriptive study. J Am Acad Dermatol. (2020) 82:499-501. doi: 10.1016/j.jaad.2019.08.006

50. Vodrahalli K, Daneshjou R, Novoa RA, Chiou A, Ko JM, Zou J. TrueImage: a machine learning algorithm to improve the quality of telehealth photos. Pac Symp Biocomput. (2021) 26:220-31. doi: 10.1142/9789811232701_0021

51. Wang MZ, Comfere NI, Murphree DH. Deep learning for automating the organization of institutional dermatology image stores. Annu Int Conf IEEE Eng Med Biol Soc. (2019) 2019:4479-82. doi: 10.1109/EMBC.2019.8857086

52. Uppal SK, Beer J, Hadeler E, Gitlow H, Nouri K. The clinical utility of teledermoscopy in the era of telemedicine. Dermatol Ther. (2021) 34:e14766. doi: 10.1111/dth.14766

53. Veronese F, Branciforti F, Zavattaro E, Tarantino V, Romano V, Meiburger KM, et al. The role in teledermoscopy of an inexpensive and easy-to-use smartphone device for the classification of three types of skin lesions using convolutional neural networks. Diagnostics. (2021) 11:451. doi: 10.3390/diagnostics11030451

54. Jain A, Way D, Gupta V, Gao Y, de Oliveira Marinho G, Hartford $\mathrm{J}$, et al. Development and assessment of an artificial intelligence-based tool for skin condition diagnosis by primary care physicians and nurse practitioners in teledermatology practices. JAMA Netw Open. (2021) 4:e217249. doi: 10.1001/jamanetworkopen.2021.7249

55. Saleh J, Dahiya M. Social media trends in dermatology, dermatopathology, and pathology publications: the social construction of medical subdisciplines. J Cutan Pathol. (2020) 47:601-5. doi: 10.1111/cup.13680

56. Galamgam J, Jia JL. "Accutane Check": Insights into youth sentiment toward isotretinoin from a TikTok trend. Pediatr Dermatol. (2021) 38:9801. doi: $10.1111 /$ pde. 14660

57. Alhayaza G, Chisti M, Binamer Y. The impact of social media on dermatologists and in captivating their patients; a cross-sectional study. $J$ Dermatolog Treat. (2021) 11:1-24. doi: 10.1080/09546634.2021.1940809

58. Viola KV, Tolpinrud WL, Gross CP, Kirsner RS, Imaeda S, Federman DG. Outcomes of referral to dermatology for suspicious lesions: implications for teledermatology. Arch Dermatol. (2011) 147:556-60. doi: 10.1001/archdermatol.2011.108

59. Armstrong AW, Kwong MW, Ledo L, Nesbitt TS, Shewry SL. Practice models and challenges in teledermatology: a study of collective experiences from teledermatologists. PLOS ONE. (2011) 6:e28687. doi: 10.1371/journal.pone.0028687

60. Tensen E, van der Heijden JP, Jaspers MWM, Witkamp L. Two decades of teledermatology: current status and integration 
in national healthcare systems. Curr Dermatol Rep. (2016) 5:96-104. doi: 10.1007/s13671-016-0136-7

61. Lee KJ, Finnane A, Soyer HP. Recent trends in teledermatology and teledermoscopy. Dermatol Pract Concept. (2018) 8:21423. doi: $10.5826 / \mathrm{dpc} .0803 \mathrm{a} 013$

62. Wang RH, Barbieri JS, Nguyen HP, Stavert R, Forman HP, Bolognia JL, et al. Group for research of policy dynamics in dermatology. Clinical effectiveness and cost-effectiveness of teledermatology: where are we now, and what are the barriers to adoption? J Am Acad Dermatol. (2020) 83:299307. doi: 10.1016/j.jaad.2020.01.065

63. Keleshian V, Ortega-Loayza AG, Tarkington P. Incidental skin malignancies in teledermatology and in-person cohorts in the Veterans Affairs Health System. J Am Acad Dermatol. (2017) 77:965-6. doi: 10.1016/j.jaad.2017.01.027

64. Hekler A, Kather JN, Krieghoff-Henning E, Utikal JS, Meier F, Gellrich FF, et al. Effects of label noise on deep learning-based skin cancer classification. Front Med. (2020) 7:177. doi: 10.3389/fmed.2020.00177

65. Jutzi TB, Krieghoff-Henning EI, Holland-Letz T, Utikal JS, Hauschild A, Schadendorf D, Sondermann W, Fröhling S, Hekler A, Schmitt M, Maron RC, Brinker TJ. Artificial Intelligence in Skin Cancer Diagnostics: The Patients' Perspective. Front Med (Lausanne). 2020 Jun 2;7:233. doi: 10.3389/fmed.2020.00233

66. Du AX, Emam S, Gniadecki R. Review of Machine Learning in Predicting Dermatological Outcomes. Front Med (Lausanne). (2020) 7:266. doi: $10.3389 /$ fmed.2020.00266
67. Schäfer Z, Mathisen A, Svendsen K, Engberg S, Rolighed Thomsen T, Kirketerp-Møller K. Toward Machine-Learning-Based Decision Support in Diabetes Care: A Risk Stratification Study on Diabetic Foot Ulcer and Amputation. Front Med (Lausanne). (2021) 7:601602. doi: 10.3389/fmed.2020.601602

Conflict of Interest: The authors declare that the research was conducted in the absence of any commercial or financial relationships that could be construed as a potential conflict of interest.

Publisher's Note: All claims expressed in this article are solely those of the authors and do not necessarily represent those of their affiliated organizations, or those of the publisher, the editors and the reviewers. Any product that may be evaluated in this article, or claim that may be made by its manufacturer, is not guaranteed or endorsed by the publisher.

Copyright (c) 2021 Mahmood, Bendayan, Ghazawi and Litvinov. This is an openaccess article distributed under the terms of the Creative Commons Attribution License (CC BY). The use, distribution or reproduction in other forums is permitted, provided the original author(s) and the copyright owner(s) are credited and that the original publication in this journal is cited, in accordance with accepted academic practice. No use, distribution or reproduction is permitted which does not comply with these terms. 